\title{
Design of Gain-Clamped Doped-Fiber Amplifiers for Optimal Dynamic Performance
}

\author{
Alberto Bononi and Lorenzo Barbieri
}

\begin{abstract}
This paper provides a detailed analysis of gainclamped doped-fiber amplifiers and design guidelines in a wavelength division multiplexed (WDM) networking environment. A simple dynamic model of the doped-fiber amplifier allows us to derive explicit expressions for the small-signal response, which help identify and optimize the most critical parameters for best dynamic performance. The most important parameter is the pump power, which should be chosen 1-2 dB's above its required open-loop value, with all channels present, for the required signal gain. In an all-optical networking scenario with input power per channel as high as $-3 \mathrm{dBm}$ the required pump power may well exceed $20 \mathrm{dBm}$. Thus optimization of other parameters such as laser wavelength and loop loss are important. For best dynamic performance either the loop loss should be extremely small, implying a very large laser flux, or the laser gain variation in response to a perturbation should be large. Accordingly, the laser wavelength should be placed either close to the unity-gain region of the clamped gain profile, or at its peak. Finally, the small signal model for a chain of clamped amplifiers is provided, and it is shown that long chains are vulnerable to low-frequency input signal perturbations.
\end{abstract}

Index Terms - Doped-amplifier gain dynamics, erbium-doped fiber amplifier (EDFA), gain-clamping.

\section{INTRODUCTION}

D OPED-FIBER amplifiers (DFA's) for wavelength division multiplexed (WDM) systems have a nonflat gainversus-wavelength profile, which greatly varies because of saturation when the input power levels are large. In the design of optically amplified links for WDM applications, in which the number and the power level of the input channels may vary randomly in time as in a networking scenario, it is thus essential to stabilize the amplifier gain profile.

Several feedback control techniques have been proposed in the literature, all more or less explicitly aiming at stablizing the average inversion of the DFA.

A first class uses some optical measure of inversion at the output of the amplifier to produce an error signal which electrically controls the power of either the pump or of an extra control input laser source [1]-[5].

A second class uses an all-optical feedback lasing signal sustained by the amplifier itself, which clamps the average inversion and thus the gain to the desired level [6]-[8]. The technique is known as gain clamping. The feedback is either

Manuscript received August 3, 1998. This work was supported in part by the European Community under INCO-DC project 950959 "DAWRON," and also in part by a grant from CSELT.

The authors are with the Dipartimento di Ingegneria dell'InformazioneUniversità di Parma, Parma I-43100 Italy.

Publisher Item Identifier S 0733-8724(99)05587-5. obtained by forming a feedback fiber loop, effectively implementing a fiber ring laser (loop configuration), or by placing fiber gratings, acting as mirrors only at the laser wavelength, at the active fiber ends (straight-line configuration). The laser plays here the role of the extra control input laser source of the previous class. Gain clamping has also been successfully applied to semiconductor optical amplifiers [9], [10] which notoriously suffer much more than DFA's from saturationinduced gain crosstalk in WDM systems.

While most of the above references deal with the steadystate analysis of gain-clamped amplifiers, a great deal of studies on their transient gain dynamics has recently appeared in the context of all-optical networks [11]-[15].

This paper addresses the design of gain-clamped doped-fiber amplifiers, utilizing the simplified dynamic model of the openloop amplifier introduced in [16], [17]. The model essentially coincides with the one in [11], where a single section is used for the doped fiber. Such a simple model allows an easy interpretation of gain clamping, and a simple study of its dynamics. System theory techniques are used to find small signal transfer functions leading to simple selection criteria for pump power, laser wavelength and loop loss for optimal dynamic performance. Also, a small signal model is developed for chains of gain-clamped amplifiers.

The paper is organized as follows. Section II introduces the dynamic nonlinear model of the gain-clamped amplifier in loop configuration. Section III deals with its steady-state analysis. Section IV introduces the design criteria for optimal dynamic performance, and Sections V, VI derive its linearized model, providing a clear picture of the dynamics inside the gainclamped DFA, and providing explicit expressions and selection criteria of the key dynamic parameters for the optimization of both isolated clamped DFA's and chains of them. Section VII summarizes the main findings. Appendix A extends the key equations to the straight-line configuration, while Appendix B defines the range of applicability of the linear model.

\section{MODEL}

The gain-clamped amplifier under study is shown in Fig. 1. It is composed of a single-section DFA, with a piece of standard fiber feeding part of the output to the DFA input. We will concentrate on the analysis of such loop configuration, although a straight-line configuration is also possible, and pertinent results discussed in Appendix A.

The positive optical feedback in the figure causes instability, and if the DFA gain is initially larger than the loop loss, 


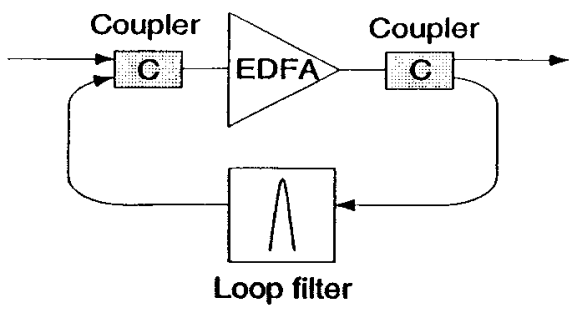

Fig. 1. Gain-clamped amplifier scheme in loop configuration.

the system starts oscillating at the wavelength selected by the narrow-band loop filter centered at wavelength $\lambda_{l}$.

In the assumption of a two-level system for the dopant ions and an homogeneously broadened gain spectrum, the DFA can be modeled as a nonlinear dynamic system with a single state variable, namely its total number of excited ions $r$, called the reservoir [16]. If $r_{\max }$ is the total number of ions in the DFA, the normalized reservoir $x \triangleq r / r_{\max }$ represents the average fraction of excited ions in the DFA, known as average inversion. The update equation for the reservoir, including self-saturation induced by the amplified spontaneous emission (ASE) noise is [17], [11]

$$
\begin{aligned}
\dot{r}(t)= & \sum_{j \in\{\mathcal{S}, p, l\}} Q_{j}(t)\left\{1-G_{j}(r(t))\right\} \\
& -\frac{r(t)}{\tau}-Q_{\mathrm{ASE}}(r(t))
\end{aligned}
$$

where $\tau$ is the fluorescence lifetime $Q_{j}, j \in \mathcal{S} \triangleq\{1 \ldots N\}$ are the input WDM signal fluxes [photons/s] at wavelengths $\lambda_{j}, Q_{p}$ the input pump flux, $Q_{l}$ the input laser flux $Q_{\mathrm{ASE}}(r)$ is the output ASE flux, a function of the average inversion in the approximation of constant inversion [18], [17]; $G_{j}(r)=$ $e^{B_{j} r-A_{j}}$ is the gain at wavelength $\lambda_{j}$ where $B_{j}$ and $A_{j}$ are nondimensional wavelength dependent coefficients given by $A_{j}=\rho \Gamma_{j} \sigma_{j}^{a} L B_{j}=\Gamma_{j}\left(\sigma_{j}^{e}+\sigma_{j}^{a}\right) / A_{\text {eff }}$, where $\rho$ is the erbium concentration, $A_{\text {eff }}$ the core effective area, $L$ the DFA length, $\sigma_{j}^{e}$ and $\sigma_{j}^{a}$ the emission and absorption cross sections at $\lambda_{j}$, and $\Gamma_{j}$ the overlap factor.

We interpret (1) as the balance between the amplifier's input and output photon fluxes, being the net contribution of that balance the reservoir's variation per unit time.

We then account for the optical feedback by writing the laser input flux as a delayed and attenuated version of the output flux, to which the ASE term $Q_{l, \mathrm{ASE}}$ passed by the feedback filter is added

$$
Q_{l}(t)=\alpha\left\{Q_{l}\left(t-\tau_{l}\right) G_{l}\left(r\left(t-\tau_{l}\right)\right)+Q_{l, \mathrm{ASE}}\left[r\left(t-\tau_{l}\right)\right]\right\}
$$

being $\tau_{l}$ the loop propagation delay and $0 \leq \alpha \leq 1$ the loop attenuation, both at laser wavelength $\lambda_{l}$.

Equations (1)-(2) form the dynamic model of the gainclamped amplifier. The number of state variables is now two, the second being the laser flux $Q_{l}(t)$.

\section{STEAdy-STATE ANALYSiS}

We first give an intuitive explanation of gain clamping. Fig. 2 gives an example of the DFA gain in $\mathrm{dB}, 4.34\left(B_{j} r-\right.$

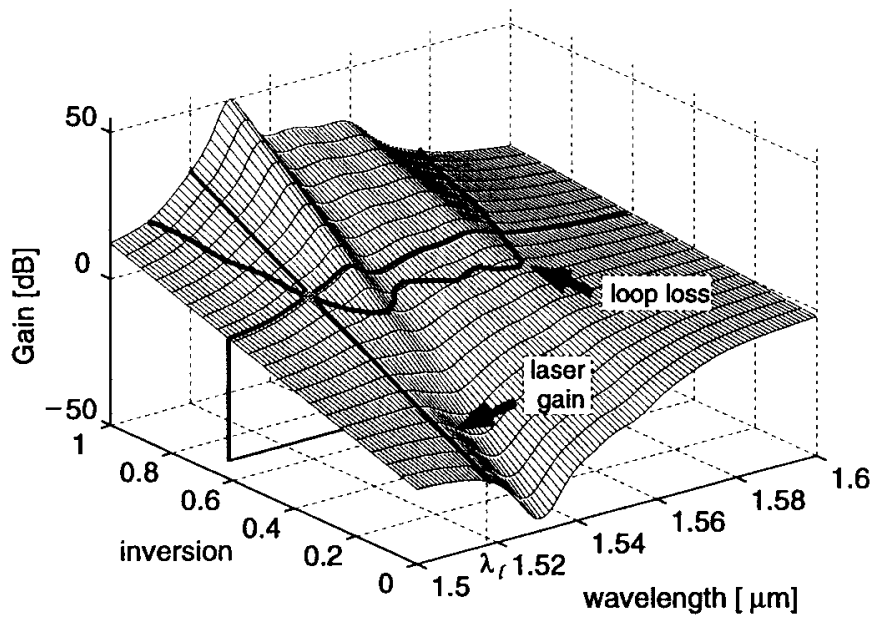

Fig. 2. Amplifier gain in $\mathrm{dB}$ versus average inversion $x \triangleq r / r_{\max }$ and wavelength.

$A_{j}$ ), plotted against wavelength and inversion. For a fixed value of the reservoir $r$, or equivalently of inversion $x$, we have the well-known gain-versus-wavelength profile due to the wavelength dependence of the coefficients $A_{j}$ and $B_{j}$. It is the variation of $x$ caused by the input power variations that causes the undesired profile changes. The $\mathrm{dB}$ gain has a linear dependence on $r$, i.e., the surface in Fig. 2 is composed of straight lines with slope depending on wavelength. The optical feedback fixes the average inversion and thus the gain profile to a desired value as follows: the loop filter passes only wavelength $\lambda_{l}$, i.e., selects the straight line corresponding to the laser gain shown on the surface. The horizontal contour line on the surface marks the level corresponding to the loop loss at wavelength $\lambda_{l}$ (the loop loss at all other wavelengths is infinity). The laser flux grows until its gain equals the loop loss, thus fixing the desired inversion. The desired inversion can be changed by either changing the loop loss for fixed $\lambda_{l}$ (thus moving along the laser gain line), or by changing $\lambda_{l}$ for fixed loss (thus moving along the loop loss contour). The equilibrium point at the intersection of the loop loss contour and the laser gain line is stable. In fact, if some channels are dropped, less reservoir ions are consumed, $x$ tends to increase, and so does the laser gain and the laser flux, which grows to consume the excess reservoir ions and brings $x$ back to its clamped value. If some channels are added, more reservoir ions are consumed, $x$ tends to decrease, and so does the laser gain and the laser flux, which consumes less reservoir ions and brings $x$ back to its clamped value.

Analytically, the system steady state is obtained by letting $\dot{r}(t)=0$ in (1) and $Q_{l}(t)=Q_{l}\left(t-\tau_{l}\right)$ in (2). As a result we get:

$$
\begin{aligned}
& r_{s s}=\tau\left\{\sum_{j \in\{\mathcal{S}, p, l\}} Q_{j}\left[1-G_{j}^{s s}\right]-Q_{\mathrm{ASE}}\left(r_{s s}\right)\right\} \\
& Q_{l}^{s s}\left[1-\alpha \cdot G_{l}^{s s}\right]=\alpha \cdot Q_{l, \mathrm{ASE}}\left(r_{s s}\right) .
\end{aligned}
$$

where $G_{j}^{s s}$ is shorthand for $G_{j}\left(r_{s s}\right)$. If the ASE term in the feedback filter bandwidth is much smaller than the steady state 
laser flux, the second equation in (3b) can be approximated as

$$
1-\alpha \cdot G_{l}^{s s}=0
$$

which gives an explicit expression for the steady state reservoir

$$
r_{s s}=\frac{\ln 1 / \alpha+A_{l}}{B_{l}} .
$$

Equation (4) is the well-known Barkhausen criterion for steady state oscillation in a feedback noiseless system [19]. ${ }^{1}$ Since the ASE power in the feedback filter bandwidth can be made small enough by appropriately choosing the filter bandwidth, in the following we will use (5) to get the steady-state reservoir solution. Note that (5) is the equation of the loop loss contour shown in Fig. 2.

In order to get physically acceptable solutions, the laser flux must be nonnegative. From (3a) and (4), we get

$$
Q_{l}^{s s}=\frac{\sum_{j \in\{\mathcal{S}, p\}} Q_{j}\left[1-G_{j}^{s s}\right]-\frac{r_{s s}}{\tau}-Q_{\mathrm{ASE}}\left(r_{s s}\right)}{1 / \alpha-1}
$$

so that the condition $Q_{l}^{s s} \geq 0$ gives:

$$
Q_{p}\left[1-G_{p}^{s s}\right] \geq \sum_{j \in \mathcal{S}} Q_{j}\left[G_{j}^{s s}-1\right]+\frac{r_{s s}}{\tau}+Q_{\mathrm{ASE}}\left(r_{s s}\right)
$$

which we interpret either as a lower bound on pump flux or as an upper bound on input fluxes [21]. With the sign of equality, (7) represents a hyperplane bounding the limit values of the variables $Q_{p}$ and $\left\{Q_{j}\right\}, j \in \mathcal{S}$. For instance, given the input fluxes $\left\{Q_{j}\right\}$ and the desired reservoir $r_{S S}$, (7) with the equality sign gives the lower bound on pump $Q_{p}^{L}$, i.e., the pump flux needed in an open-loop (nonclamped) DFA to reach the desired inversion. Using a pump flux $Q_{p}>Q_{p}^{L}$, the net laser flux in (6) can be rewritten as

$$
Q_{l}^{s s}(1 / \alpha-1)=\left(Q_{p}-Q_{p}^{L}\right)\left(1-G_{p}^{s s}\right) .
$$

which is independent of laser wavelength.

Let us now consider a numerical example, to which we will often refer. The erbium DFA (EDFA) parameters used are: pump wavelength $\lambda_{p}=1480 \mathrm{~nm}$; fluorescence time $\tau=10.5$ ms; erbium concentration $\rho=1.14 \times 10^{24}$ ions $/ \mathrm{m}^{3}$; overlap factor $\Gamma=0.5$; effective core area $A_{\text {eff }}=5.12 \times 10^{-12}$ $\mathrm{m}^{2}$; EDFA length $L=35 \mathrm{~m}$; cross-section values taken from fitted Lorentzian curves in [[22], Table 4.2, p. 299] with $\sigma_{\max }^{e}=5.8 \times 10^{-25} \mathrm{~m}^{2}$ and $\sigma_{\max }^{a}=5.3 \times 10^{-25} \mathrm{~m}^{2}$. The WDM system is composed of eight channels with equal input power per channel $P_{\mathrm{in} / \mathrm{ch}}$ at frequencies chosen according to the ITU-T standard between $192.8 \mathrm{THz}(1554.9 \mathrm{~nm})$ and 193.5 THz $(1549.3 \mathrm{~nm})$, with $100 \mathrm{GHz}(0.8 \mathrm{~nm})$ spacing.

Fig. 3 shows the lower bound on pump power $P_{p}^{L} \triangleq h \nu_{p} Q_{p}^{L}$ versus normalized steady-state reservoir, with input power per channel $P_{\mathrm{in} / \mathrm{ch}}$ as a parameter when all eight WDM channels are present. Such a minimum pump is the one needed by the open-loop amplifier to guarantee the required gain level and profile with all channels present, and thus to ensure the

\footnotetext{
${ }^{1}$ The oscillation condition on the signal phase is neglected here since the lasing modes form a continuum, because the loop delay $\tau_{l}$ is usually more than six orders of magnitude larger than the mode period.
}

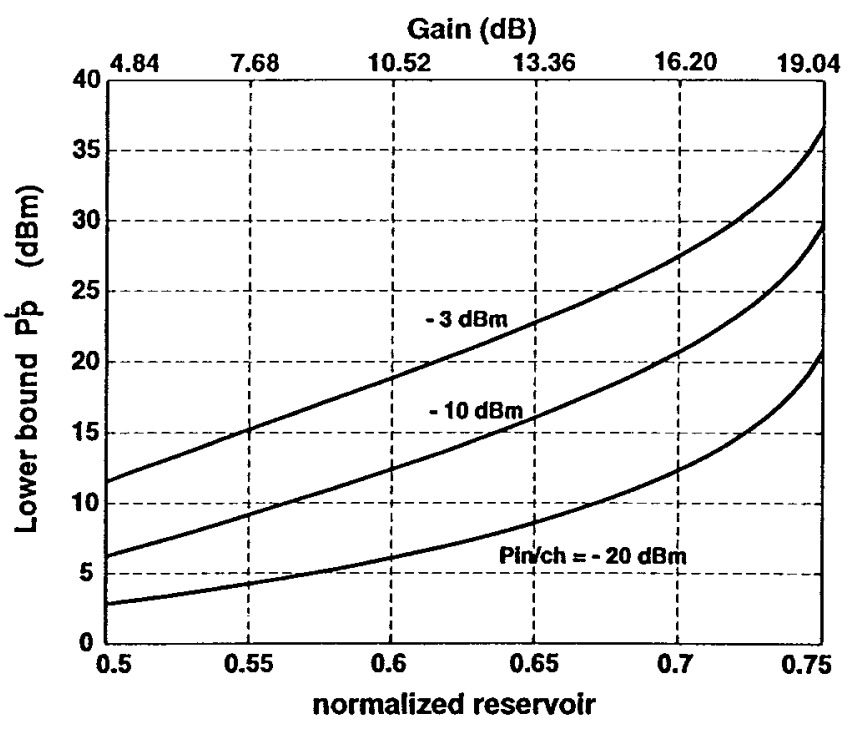

Fig. 3. Lower bound on pump power $P_{p}^{L}$ versus average inversion $x$, with input power per channel as a parameter.

existence of the laser oscillation in all other possible static configurations of the WDM system. The reservoir fixes the gain profile and level, which in the middle of the WDM comb has the $\mathrm{dB}$ values indicated in the upper "Gain" axis. We observe that for a typical interamplifier loss from 10 to $15 \mathrm{~dB}$ the average inversion is around 0.63 . The pump lower bound is below $10 \mathrm{~dB}$ for signal levels of $-20 \mathrm{dBm} / \mathrm{ch}$, below 16 $\mathrm{dB}$ for $-10 \mathrm{dBm} / \mathrm{ch}$, and as high as $23 \mathrm{~dB}$ for a typical value for all-optical networks such as $-3 \mathrm{dBm} / \mathrm{ch}$. This means that a very large pump value is already required for open-loop EDFA's to guarantee the required gain and profile.

In the next section we will show that a pump a few dBs larger than the lower bound must be provided to sustain the laser oscillation even when all channels are present, in order to get a satisfactory dynamic response.

\section{Design for Optimal Dynamic Performance}

In the design of the gain-clamped amplifier, we have a constraint on the required gain level and gain-versus-wavelength profile for the WDM channels, of which we know the number and the input power level $P_{\mathrm{in} / \mathrm{ch}}$. Once the profile is stabilized by gain clamping, gain equalization can optionally be achieved by using a suitable passive optical filter [23]. Considering that the major cost is due to the large pump power in the DFA, our target is to stabilize a given gain profile, for given input channels and required gain level, by minimizing the required pump power.

Since a one-to-one relation exists between gain profile and reservoir value, system specifications fix the required value for $r_{s s}$ at steady state. Given $r_{s s}$, laser wavelength $\lambda_{l}$ and loop loss are related by the Barkhausen criterion (5). We have one degree of freedom in the choice between laser loss and wavelength.

Another degree is available with the selection of the pump power, for which a lower bound to ensure the existence of the laser oscillation with all channels present was obtained in the previous section. 
(a)

(b)

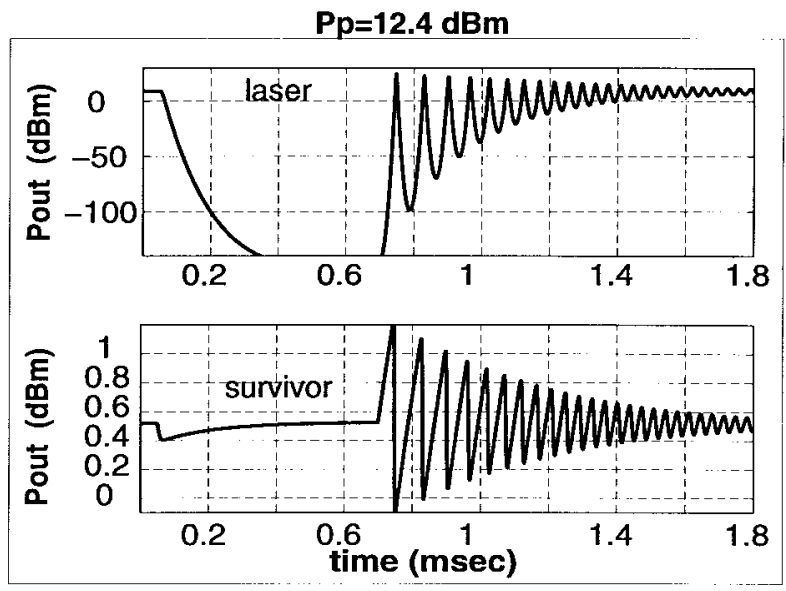

$P p=13.9 \mathrm{dBm}$
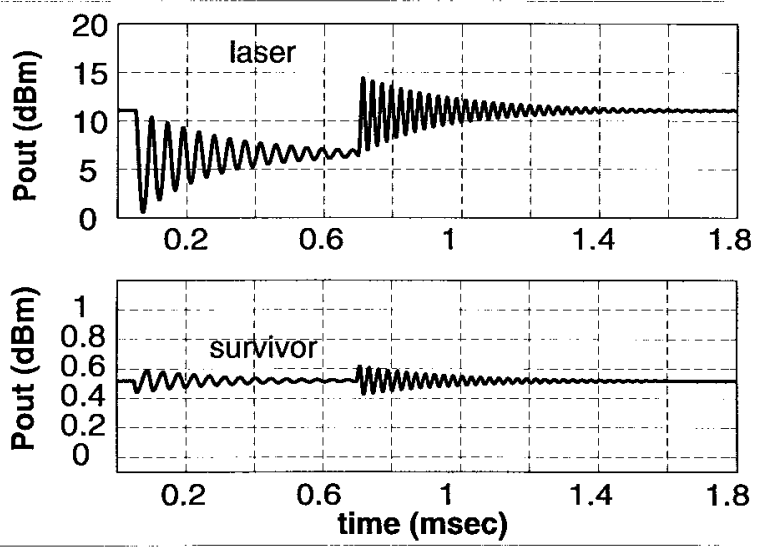

Fig. 4. (a) Laser and (b) surviving channel output power time response to a seven out of eight channel add-drop sequence, with $P_{\mathrm{in} / \mathrm{ch}}=-10 \mathrm{dBm}$, $x=0.6, \lambda_{l}=1530 \mathrm{~nm}, \tau_{l}=0.18 \mu \mathrm{s}$.

To understand the effect of choosing the pump level very close to its lower bound (see Fig. 3) on the dynamic response of the clamped amplifier, we note that in this case the laser level approaches zero. Starting with all channels present, when a channel drop occurs a long "switch on" transient takes place, giving a slow response and large overshoots on the surviving channels. This effect is shown in Fig. 4. Fig. 4(a) refers to a system with average inversion $x=0.6, P_{\mathrm{in} / \mathrm{ch}}=-10$ $\mathrm{dBm}, \lambda_{l}=1530 \mathrm{~nm}$, and pump power $P_{p}=12.4 \mathrm{dBm}$ corresponding to its lower bound. The loop delay is $\tau_{l}=$ $0.18 \mu \mathrm{s}$ corresponding to $40 \mathrm{~m}$ of fiber propagation. The figure shows a double transient: before time 0 there is only one active channel, and at time $0.05 \mathrm{~ms}$ the remaining seven channels are added. The transition is abrupt, i.e., takes place in zero time, which gives a worst-case dynamic behavior [12]. Being the pump power at its lower bound, when all channels are present the laser switches off. After some fraction of ms the laser power is negligible compared to the WDM channels and does not influence the dynamics of the surviving channel anymore. At time $0.7 \mathrm{~ms}$ the seven channels are dropped. Clearly, since the laser is essentially off, a long $(0.1 \mathrm{~ms})$ switch-on transient occurs, and large $(1.2 \mathrm{~dB})$ power excursions show up on the surviving channel, with a long ringing. It takes more than 1 ms for the system to settle to the new steady-state. Fig. 4(b) shows what happens if the pump is chosen $1.5 \mathrm{~dB}$ above the lower bound, $P_{p}=13.9 \mathrm{dBm}$. In such case an output laser oscillation $(6.5 \mathrm{dBm})$ is present at the next steady-state after the seven-channel add, so that the subsequent seven-channel drop causes a transient in the system that settles after a little more than $0.4 \mathrm{~ms}$, with a maximum power excursion on the surviving channel of only $0.1 \mathrm{~dB}$. We note that the maximum $\mathrm{dB}$-excursion is larger at the drop, with ringing (also known as relaxation oscillations [24]) at higher frequency with respect to the add, but with comparable decay rates [15].

From this we learn that extra pump is a key factor in the dynamic step response of the system. Other important factors are the gain on the surviving channel (or equivalently the average inversion), the input power per channel, and the laser wavelength. We repeated the add-drop experiment by varying the above parameters, and recorded the maximum dB-power excursion at the drops. The results are summarized in the contour plots of Fig. 5. We see for instance that the laser wavelength $\lambda_{l}=1530 \mathrm{~nm}$ gives much lower excursions than $\lambda_{l}=1570 \mathrm{~nm}$, and that with at least $1.5 \mathrm{~dB}$ extra pump and $\lambda_{l}=1530 \mathrm{~nm}$ the maximum power excursion can be kept below $0.2 \mathrm{~dB}(0.4 \mathrm{~dB})$ for input power per channel $P_{\mathrm{in} / \mathrm{ch}}=-10 \mathrm{dBm}\left(P_{\mathrm{in} / \mathrm{ch}}=-3 \mathrm{dBm}\right)$ in our eight channel WDM system, for signal gains up to $18 \mathrm{~dB} .^{2}$.

To get an understanding of the key system parameters determining the dynamic response of the clamped amplifier, we now perform a small-signal analysis, leading to simple approximations of the dynamic response. For example, we give in Appendix B an analytic approximation of the above contour plots.

\section{SMALl-Signal ANALYSiS}

The nonlinear system described by (1) and (2) has two state variables, namely, the reservoir $r$ and the laser input flux $Q_{l}$, which reach steady state under continuous-wave $(\mathrm{CW})$ signals. In this section we slightly perturb the state equilibrium point to obtain explicit expressions of the system response to any signal/pump perturbation which causes a small perturbation of the state. We carry on the analysis neglecting ASE, as the clamped DFA is normally saturated by the laser flux [15]. The accuracy of the linear model is discussed in detail in Appendix B.

\section{A. Reservoir and Laser Filters}

Let the perturbed reservoir be $r(t) \triangleq r_{s s}+\Delta r(t)$ and the perturbed input fluxes be $Q_{j}(t) \triangleq Q_{j}^{s s}+\Delta Q_{j}(t), j \in\{\mathcal{S}, p, l\}$. Assuming that $\max _{j \in\{\mathcal{S}, p, l\}}\left\{B_{j}\right\} \Delta r(t) \ll 1$, we approximate the gain as $G_{j}(r(t))=G_{j}^{s s} e^{B_{j} \Delta r(t)} \cong G_{j}^{s s}\left[1+B_{j} \Delta r(t)\right]$. Using the above expressions in (1) we obtain

$$
\dot{\Delta} r(t)=-\frac{\Delta r(t)}{\tau_{o}}+\sum_{j \in \mathcal{F}} \Delta Q_{j}(t)\left(1-G_{j}^{s s}\left(1+B_{j} \Delta r(t)\right)\right)
$$

where $\mathcal{F}$ is the set of input fluxes $(\mathcal{F}=\{\mathcal{S}, p\}$ for the unclamped DFA, and $\mathcal{F}=\{\mathcal{S}, p, l\}$ for the clamped DFA),

\footnotetext{
${ }^{2}$ With our EDFA parameters, signal gains of $[6,8,10,12,14,16,18] \mathrm{dB}$ correspond to average inversions $[0.52,0.55,0.59,0.62,0.66,0.69,0.73]$
} 

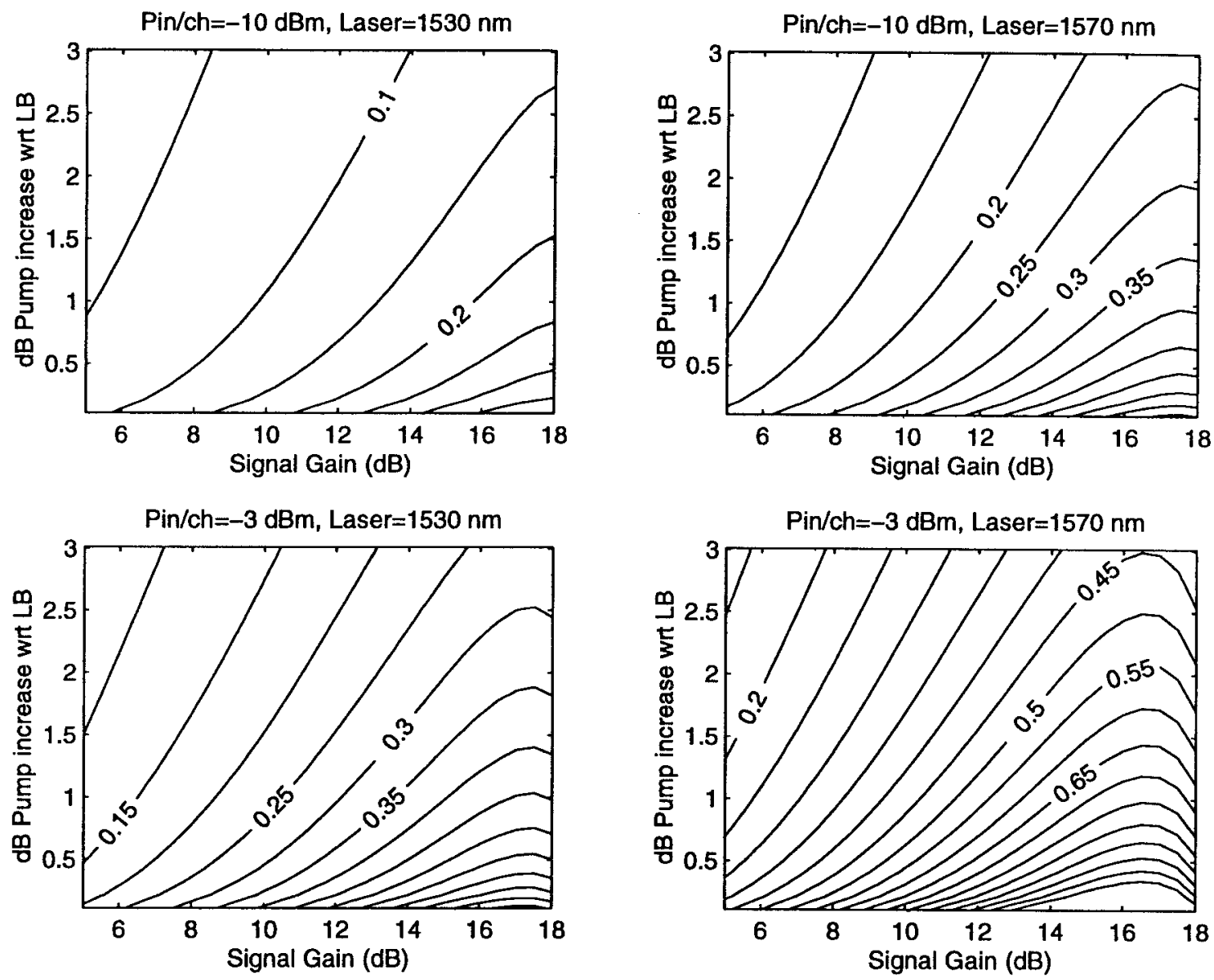

Fig. 5. Contour plots of maximum dB-excursion on surviving channel for a seven out of eight channel drop. $Y$-axis: $Q_{p} / Q_{p}^{L}$ (dB); $X$-axis: surviving signal gain $(\mathrm{dB})$. Round-trip delay $\tau_{l}=0.18 \mu \mathrm{s}$. Contour levels spaced by $0.05 \mathrm{~dB}$.

and

$$
\frac{1}{\tau_{o}} \triangleq \frac{1}{\tau}+\sum_{j \in \mathcal{F}} Q_{j}^{s s} G_{j}^{s s} B_{j}
$$

Neglecting $B_{j} \Delta r$ with respect to one, we can take the Laplace transform of both sides of (9) to get a linear relation between the Laplace transform $\Delta R(s)$ of $\Delta r(t)$ and the transform $\Delta Q_{j}(s)$ of $\Delta Q_{j}(t)$

$$
\left(s+\frac{1}{\tau_{o}}\right) \Delta R(s)=\sum_{j \in \mathcal{F}}\left(1-G_{j}^{s s}\right) \Delta Q_{j}(s) .
$$

Equation (11) can be rewritten as

$$
\Delta R(s)=K(s) H_{o}(s)
$$

where $K(s)=\Sigma_{j \in \mathcal{F}}\left(1-G_{j}^{s s}\right) \Delta Q_{j}(s)$, and

$$
H_{o}(s) \triangleq \frac{1}{s+\frac{1}{\tau_{o}}}
$$

is the transfer function of a lowpass filter of 3-dB bandwidth $1 / \tau_{o}$ and DC value $\tau_{o}$. In the absence of optical feedback, the open-loop filter $H_{o}(j \omega)$ passes the low-frequency components of the signal/pump flux variations to the reservoir. The openloop 3-dB bandwidth increases with the input fluxes, i.e., with the saturation level, and for large fluxes is almost independent of the fluorescence time $\tau$ [25].

When optical feedback is present, $\Delta Q_{l}(s)$ is a function of $\Delta R(s)$. To find such function, we start from (2), with the previous expansion of the gain term, to get the laser input flux variation

$$
\begin{aligned}
\Delta Q_{l}(t)= & \Delta Q_{l}\left(t-\tau_{l}\right)\left(1+B_{l} \Delta r\left(t-\tau_{l}\right)\right) \\
& +Q_{l}^{s s} B_{l} \Delta r\left(t-\tau_{l}\right) .
\end{aligned}
$$

Again neglecting $B_{l} \Delta r$ with respect to one, taking the Laplace transform of both sides of this equation, and using a firstorder Padé rational approximation for the delay term $e^{-\tau_{l} s} \simeq$ $\left(1-\tau_{l} s / 2\right) /\left(1+\tau_{l} s / 2\right)$ [26] we get

$$
\Delta Q_{l}(s)=\Delta R(s) \frac{Q_{l}^{s s} B_{l}}{\tau_{l} s}\left(1-\frac{\tau_{l}}{2} s\right) .
$$

Using (15) in (11) and the fact that $G_{l}^{s s}=1 / \alpha$, we get an expression for $\Delta R(s)$ of the same form as (12) where $H_{o}(s)$ is replaced by the closed-loop transfer function

$$
H_{c}(s) \triangleq \frac{s}{s^{2}+\frac{1}{\tau_{c}} s+\left(\frac{1}{\alpha}-1\right) \frac{Q_{l}^{s s} B_{l}}{\tau_{l}}}
$$


(a)

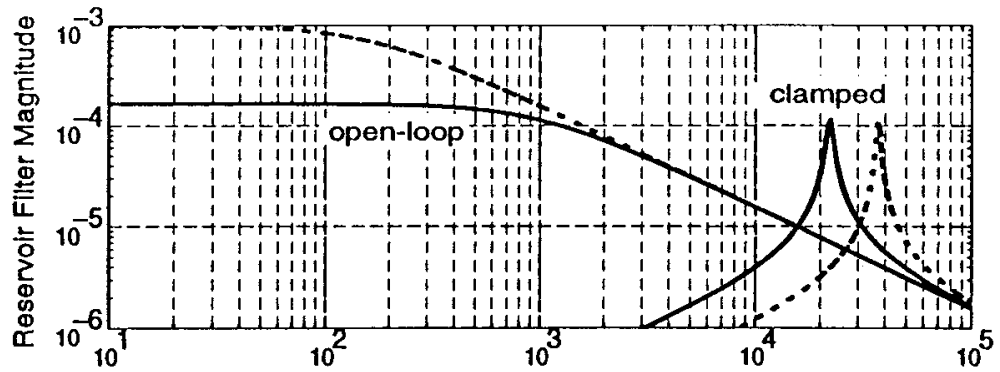

(b)

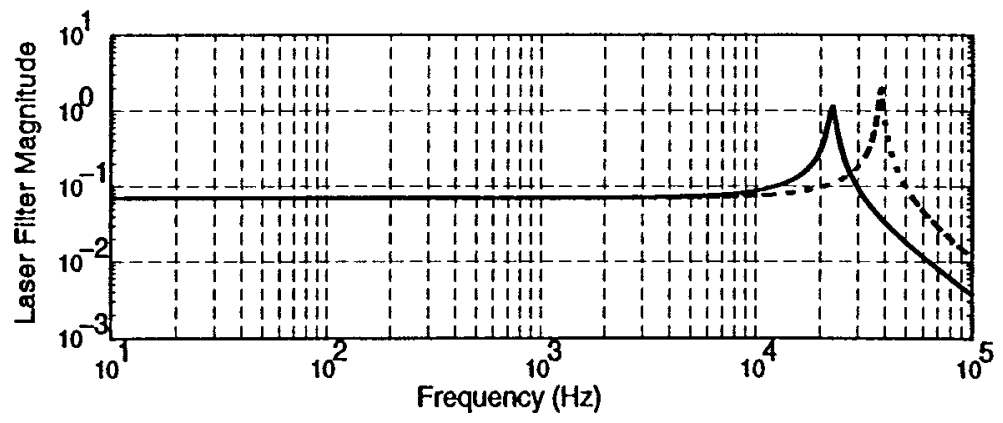

Fig. 6. (a) Reservoir filter magnitude, clamped $\left|H_{c}(j \omega)\right|$ and open-loop $\left|H_{o}(j \omega)\right|$ and (b) laser filter magnitude $\left|H_{l}(j \omega)\right|$. Data: $x=0.6, \tau_{l}=0.18 \mu \mathrm{s}$, $\lambda_{l}=1530 \mathrm{~nm}, P_{\mathrm{in} / \mathrm{ch}}=-10 \mathrm{dBm}$, pump power $13.9 \mathrm{dBm}$ for clamped DFA and $12.4 \mathrm{dBm}$ for unclamped DFA. Solid line: eight channels at steady-state; dashed: 1 channel.

where

$$
\frac{1}{\tau_{c}} \triangleq\left[\frac{1}{\tau}+\sum_{j \in\{\mathcal{S}, p\}} Q_{j}^{s s} G_{j}^{s s} B_{j}\right]+\left(\frac{1}{\alpha}+1\right) \frac{Q_{l}^{s s} B_{l}}{2} .
$$

The denominator in (16) is a second-order polynomial and can be written as [19], [20]: $s^{2}+2 \xi \Omega_{n} s+\Omega_{n}^{2}=\left(s+s_{1}\right)\left(s+s_{2}\right)$ where

$$
\Omega_{n} \triangleq \sqrt{\left(\frac{1}{\alpha}-1\right) \frac{Q_{l}^{s s} B_{l}}{\tau_{l}}}
$$

is the natural system angular frequency, $\xi \triangleq \frac{1}{2 \tau_{s} \Omega_{0}}$ the damping factor, and $s_{1}$ and $s_{2}$ the roots, with $\left|s_{1}\right|=\left|s_{2}\right|=$ $\Omega_{n}$. The underdamped case $\xi<1$ gives oscillations in the natural system response and complex conjugate roots $s_{1,2}=-\Gamma \pm j \Omega$, where $\Gamma=\frac{1}{2 \tau_{c}}$ is the decay rate and $\Omega=\Omega_{n} \sqrt{1-\xi^{2}}$ the relaxation-oscillation angular frequency. When $\xi<1, H_{c}(j \omega)$ is a bandpass selective filter, taking peak value $\tau_{c}$ at $\omega=\Omega_{n}$, and with 3 -dB bandwidth $1 / \tau_{c}$.

Fig. 6(a) shows Bode plots of the magnitude of both the open-loop $H_{o}(j \omega)$ and closed-loop $H_{c}(j \omega)$ reservoir filters for the same average inversion $x=0.6, P_{\mathrm{in} / \mathrm{ch}}=-10 \mathrm{dBm}$, $\lambda_{l}=1530 \mathrm{~nm}$ (loop loss $11.85 \mathrm{~dB}$ ), $\tau_{l}=0.18 \mu \mathrm{s}$, closedloop pump $P_{p}=13.9 \mathrm{dBm}$. To get the same inversion in the open-loop case, a pump of value $P_{p}^{L}$ is used. Solid lines correspond to a steady state with all eight channels present; dashed lines to a steady state with only one channel present. For the same inversion, the top magnitude of the open-loop filter is always larger than that of the closed-loop one, since $\tau_{o}>\tau_{c}$, although the values are comparable, and so are the 3 - $\mathrm{dB}$ bandwidths (this is not immediately clear in the log$\log$ Bode plot). Also, both filters roll off as $1 / \omega$ for large frequencies, i.e., at a rate of $-10 \mathrm{~dB} /$ decade. The closedloop filter does not transmit the low-frequency signal/pump fluctuations to the reservoir; such fluctuations are instead passed by the open-loop filter. This means for example that all the low-frequency relative intensity noise of a cheap pump can effectively be neutralized by clamping. On the other hand, no low-frequency pump overtones can be transmitted to the reservoir and hence impressed on the transiting signals for link monitoring. Moreover, when the steady-state total input power is decreased, as in the figure, the laser flux increases and so does the natural frequency, as per (18). The shift in natural frequency in the example is about $20 \mathrm{kHz}$. Such strong shift does not allow transmission of pump overtones at the natural frequency, as this is strongly input-signal dependent.

With clamping, the signal/pump low-frequency fluctuations are not transmitted to the reservoir since the laser flux takes them on. In fact, from (12) and (15), we have $\Delta Q_{l}(s)=$ $K(s) H_{l}(s)$ where the laser filter is

$$
H_{l}(s)=\frac{\left(1-\frac{\tau_{l}}{2} s\right) \frac{\Omega_{n}^{2}}{(1 / \alpha-1)}}{s^{2}+\frac{1}{\tau_{c}} s+\Omega_{n}^{2}}
$$

which corresponds to a second-order low-pass filter, as shown in Fig. 6(b).

To check the dependence of the roots on laser wavelength, Fig. 7 shows the root locus $s_{1,2}$ on the complex plane, as the laser wavelength is swept from $\lambda_{l}=1511.01 \mathrm{~nm}$ up to $\lambda_{l}=1640 \mathrm{~nm}$. The figure was obtained with all eight channels present at steady state, $P_{\mathrm{in} / \mathrm{ch}}=-10 \mathrm{dBm}$, clamped inversion $x=0.6$, loop delay $\tau_{l}=0.18 \mu$ s. Since inversion is clamped, at each point the loss changes according to (5) and the values are shown for the marked points. Two different loci are shown for pump power 18 and $13.9 \mathrm{dBm}$. We note that for loss values larger than $0.16 \mathrm{~dB}$ the roots are complex conjugate, i.e., $\xi>1$, and for reasonable loop loss larger than $1 \mathrm{~dB}\left(\lambda_{l}>1512.8\right.$ 


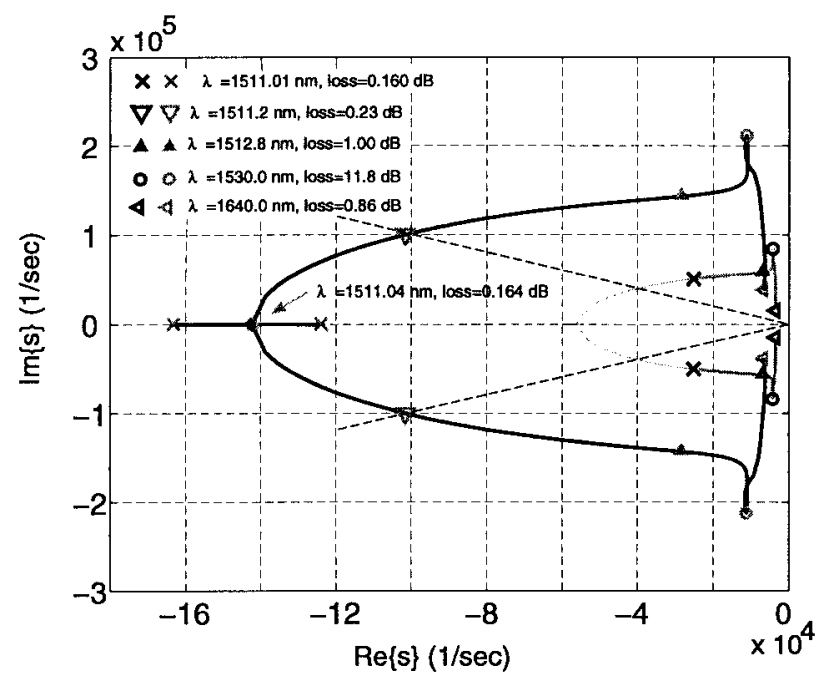

Fig. 7. Root locus $s_{1,2}$ of the small-signal characteristic equation as $\lambda_{l}$ is swept across its allowed range, for $P_{\text {in }} / \mathrm{ch}=-10 \mathrm{dBm}, x=0.6$ and two pump values: (black) $18 \mathrm{dBm}$; (gray) $13.9 \mathrm{dBm}$.

$\mathrm{nm})$ the system is strongly resonant, i.e., $\xi=\Gamma / \Omega_{n} \ll 1$, so that $\Omega \cong \Omega_{n}$. In fact, the dashed $45^{\circ}$ lines, at which real and imaginary absolute values are equal, mark the limit at which ringing becomes significant [20].

\section{B. Step Response and Choice of Laser Wavelength}

If the signals undergo an add-drop discontinuity at time zero $\Delta Q_{j}(s)=\left(\Delta Q_{j} / s\right)$, then by inverse Laplace transforming $\Delta R(s)$ the explicit reservoir variation when $\xi<1$ is obtained as

$$
\Delta r(t)=\mathcal{K} \frac{e^{-\Gamma t}}{\Omega} \sin \Omega t
$$

where $\mathcal{K}=\Sigma_{j \in\{\mathcal{S}, p\}}\left(1-G_{j}^{s s}\right) \Delta Q_{j}$.

This is a damped sinusoid, with decay rate $\Gamma$ and frequency $\Omega / 2 \pi$. From Fig. 7 we see that the fastest settling time in the step response is obtained by choosing coincident real roots, but this requires an unrealistically low loss $(0.16 \mathrm{~dB})$ at 18 $\mathrm{dBm}$ pump, and even less with a pump of $13.9 \mathrm{dBm}$.

The time behavior of $\Delta r(t)$ is important, since the $\mathrm{dB}$ power excursion $\epsilon$ on any "surviving" channel $s$ after the step variation depends linearly on it as:

$$
\epsilon \triangleq 10 \log _{10}\left\{\frac{P_{s}^{\text {out }}\left(r_{s s}+\Delta r(t)\right)}{P_{s}^{\text {out }}\left(r_{s s}\right)}\right\}=4.34 B_{s} \Delta r(t) .
$$

The maximum reservoir variation, or overshoot, is reached in such resonant systems very close to the point where the argument of the sine term is $\pi / 2: \Delta r_{\max } \cong(|\mathcal{K}| / \Omega) \exp (-(\Gamma / \Omega)(\pi / 2))$. For strongly resonant systems (i.e., for standard loop loss values larger than a few decibels) the exponential term is close to unity and $\Omega \cong \Omega_{n}$, so that

$$
\Delta r_{\max } \cong \frac{|\mathcal{K}|}{\Omega_{n}}
$$

i.e., the reservoir overshoot is roughly inversely proportional to the natural frequency.
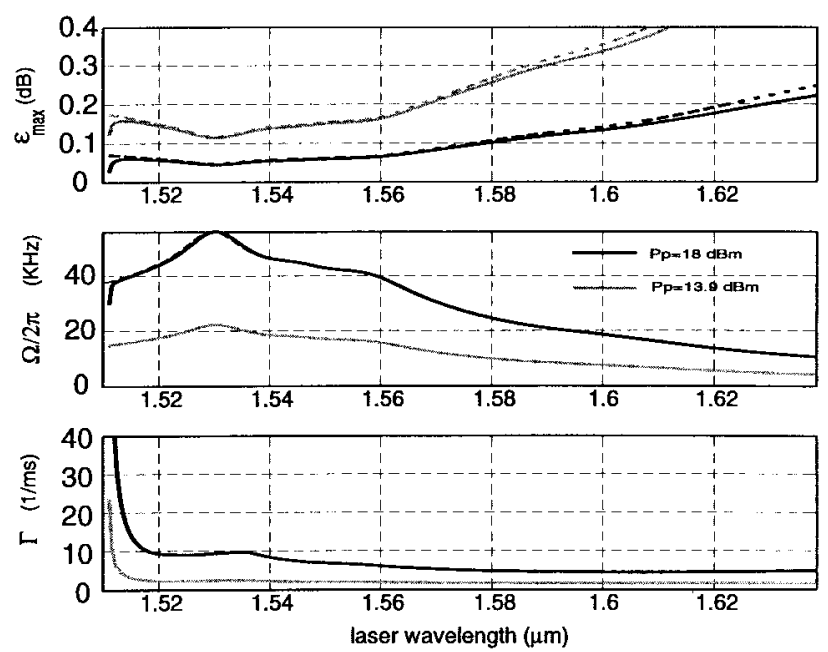

Fig. 8. Maximum dB-power excursion on surviving channel $\epsilon_{\max }$, relaxation oscillation frequency $\Omega$ and decay rate $\Gamma$ versus laser wavelength $\lambda_{l}$ for a step response to a drop of 7 out of 8 channels. Data: $P_{p}=13.9 \mathrm{dBm}$, $P_{\text {in } / \mathrm{ch}}=-10 \mathrm{dBm}, x=0.6 ; \tau_{l}=0.18 \mu \mathrm{s}$.

Using (8) and the definitions, we rewrite

$$
\begin{aligned}
\Omega_{n}= & \sqrt{\frac{B_{l}}{\tau_{l}}\left(Q_{p}-Q_{p}^{L}\right)\left(1-G_{p}^{s s}\right)} \\
\Gamma= & \frac{1}{2}\left[\left(\frac{1}{\tau}+\sum_{j \in\{\mathcal{S}, p\}} Q_{j}^{s s} G_{j}^{s s} B_{j}\right)\right. \\
& \left.+\frac{1 / \alpha+1}{1 / \alpha-1} \frac{B_{l}}{2}\left(Q_{p}-Q_{p}^{L}\right)\left(1-G_{p}^{s s}\right)\right] .
\end{aligned}
$$

This shows that $\Omega_{n}$ depends on laser wavelength only through the factor $B_{l}$, while $\Gamma$ through the factor $F \triangleq B_{l}(1 / \alpha+$ $1 / 1 / \alpha-1$. $F$ should be large for fast oscillation decay. Maximizing $B_{l}$ maximizes the laser gain variation induced by a given reservoir variation while minimizing the loss maximizes the input laser flux in photons/s as per (6): in both ways the laser reaction to the add-drop, coming from a decrease/increase of the ions consumed in the reservoir, is improved in speed.

Fig. 8 shows the dependence of the overshoot $\epsilon_{\max }, \Omega$ and $\Gamma$ on $\lambda_{l}$ for a pump of $18 \mathrm{dBm}$ (black) and 13.9 (gray). Such curves for $\Omega$ and $\Gamma$ were given implicitly in the root locus of Fig. 7. From the figures and from (23) we see that increasing the pump increases $\Omega$ (more oscillations), increases $\Gamma$ (faster decay), and consequently decreases $\epsilon$ (less overshoot), in accord with our previous observation in Fig. 4. On the graph of $\Omega$ (cf., [15, Fig. 4]) we have superposed the graph of $\Omega_{n}$ in dashed line and the two curves almost exactly overlap on most of the laser range shown, except when $\lambda_{l}$ is close to $1511 \mathrm{~nm}$, where the roots tend to merge and the system loses its high resonance. The dashed lines on the graph of $\epsilon_{\max }$ represent (21) with approximation (22), and we see that $\epsilon_{\max }$ is in practice inversely proportional to $\Omega_{n}$, and reaches its minimum where $\Omega_{n}$ has its maximum, i.e., where $B_{l}$ is maximum. This implies that at high resonance the most effective laser wavelength to minimize $\epsilon_{\max }$ for a given pump is the wavelength maximizing $B_{l}$, typically around $1530 \mathrm{~nm}$. 

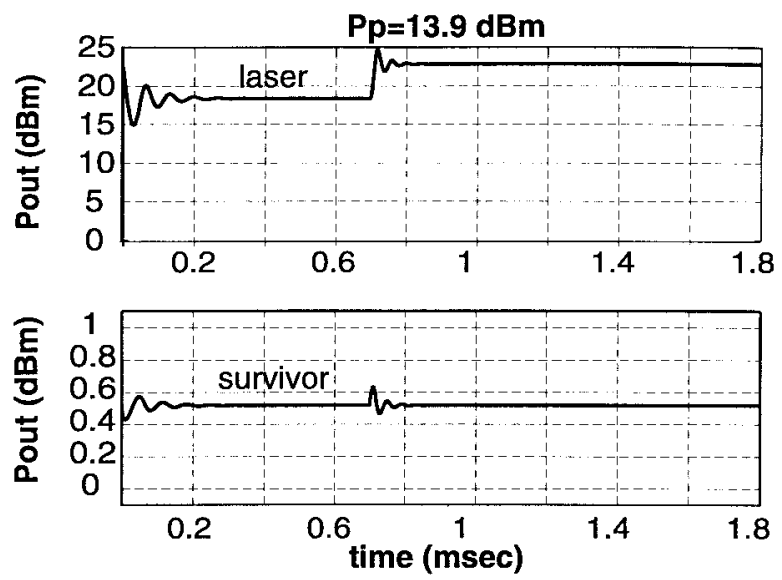

Fig. 9. Same add-drop transient as in Fig. 4, but with a laser wavelength $\lambda_{l}=1511.3 \mathrm{~nm}$, corresponding to a loop loss of $0.27 \mathrm{~dB}$.

We also note from (23) that $\Gamma$ tends to infinity when $\alpha$ tends to one, i.e., close to the critical damping and away from strong resonance. Thus, another interesting option for the laser placement is near $1511 \mathrm{~nm}$ where the loop loss is close to 1 and laser flux is largest. Although in the loop configuration such low loss is not feasible, the straight-line configuration (Appendix A) can get close to that. To test the goodness of this choice even in the large signal regime, the same double transient obtained in Fig. 4 with $\lambda_{l}=1530 \mathrm{~nm}$, was repeated in Fig. 9 for $\lambda_{l}=1511.3 \mathrm{~nm}$, where the loop loss is $0.27 \mathrm{~dB}$. We note that although $\epsilon_{\max }$ is slightly larger than in Fig. 4, still damping is much stronger, and oscillations die out more quickly.

It has been pointed out in [27] that the best noise figure is obtained for lowest input laser flux, giving maximum inversion at the input. Lowest input laser flux corresponds to largest laser loss, and thus $1530 \mathrm{~nm}$ is a good choice for both noise figure and dynamic response. However, the principal noise figure degradation in a clamped DFA comes from the WDM signal loss at the input coupler, which should therefore be minimum. According to where the laser is placed, either we can live with a large loop loss and use for example a cheap input $90 / 10$ coupler, or we must use a laser well out of band, so that a wavelength multiplexer can be used, which introduces minimum input signal loss, without too much increase in the laser loop loss. Also, note that a gain-clamped amplifier can only degrade the noise figure with respect to its open-loop counterpart, since without clamping the inversion is always larger when not all WDM channels are present (the laser consumes the extra inversion in the absence of some of the WDM channels).

Finally, a key factor for the choice of laser wavelength is spectral hole burning due to inhomogeneous broadening [15], an effect which is not captured by our model. In essence, the laser wavelength should not be too far from the signals to avoid steady state gain offsets from the desired level. In this regard, $1530 \mathrm{~nm}$ is better than $1511 \mathrm{~nm}$, although it is still very far from the signals, usually located in the 1540-1550 $\mathrm{nm}$ band. Moreover, optical filters at the EDFA output are often used to prevent the major ASE components at 1530 $\mathrm{nm}$ to propagate down a chain of amplifiers, in which case the laser from a clamped amplifier cannot be propagated and thus cannot stabilize the downstream (nonclamped) EDFA's as proposed in [12].

\section{INPUT-OUTPUT LINEAR MODEL}

We complete the linear model by characterizing the DFA as an $N \times N$ linear system, whose inputs are the WDM input flux perturbations and whose output the WDM output flux perturbations. ${ }^{3}$

For every input channel $c \in \mathcal{S}$ at steady state we have $Q_{c}^{\text {out }}=Q_{c}^{\text {in }} G_{c}$. Differentiating, we get $\Delta Q_{c}^{\text {out }}=G_{c} \Delta Q_{c}^{\text {in }}+$ $Q_{c}^{\text {in }} \Delta G_{c}$, where from Section V-A we have $\Delta G_{c}=G_{c} B_{c} \Delta r$ and $\Delta R(s)=\left(\Sigma_{j \in \mathcal{S}}\left(1-G_{j}\right) \Delta Q_{j}^{\text {in }}(s)\right) H_{R}(s)$ where the reservoir filter $H_{R}(s)$ is either open loop (13) or closed loop (16). Hence, for every input channel $c \in \mathcal{S}$ we have

$$
\begin{aligned}
\Delta Q_{c}^{\text {out }}= & G_{c} \Delta Q_{c}^{\text {in }}+H_{R}(s) Q_{c}^{\text {in }} G_{c} B_{c} \\
& \cdot\left(\sum_{j \in \mathcal{S}}\left(1-G_{j}\right) \Delta Q_{j}^{\text {in }}(s)\right) .
\end{aligned}
$$

Such equations can be written in matrix form as $[25]^{4}$

$$
\Delta Q^{\text {out }}=\underline{\underline{M}}(s) \Delta Q^{\text {in }}
$$

where $\Delta Q \triangleq\left[\Delta Q_{1} \ldots \Delta Q_{N}\right]^{T}$, and the system matrix is

$$
\underline{\underline{\boldsymbol{M}}}(s)=\underline{\underline{\boldsymbol{G}}}+H_{R}(s) \boldsymbol{Q P} \boldsymbol{P}^{T}
$$

where $\underline{\underline{G}} \triangleq \operatorname{Diag}\left[G_{1} \ldots G_{N}\right]$ is a diagonal gain matrix $\boldsymbol{Q} \triangleq\left[Q_{1}^{\mathrm{in}} G_{1} B_{1} \ldots Q_{N}^{\mathrm{in}} G_{N} B_{N}\right]^{T} \boldsymbol{P} \triangleq\left[\left(1-G_{1}\right) \ldots(1-\right.$ $\left.\left.G_{N}\right)\right]^{T}$. We note that the dependence on the complex variable $s$ of such matrix comes from the scalar $H_{R}(s)$, and that the off-diagonal elements of $\underline{M}$ are just scaled versions of $H_{R}(s)$, i.e., a perturbation on channel $j$ has the same lowpass (bandpass) effect on the reservoir and on channel $k \neq j$ in open-loop (clamped) configuration.

On the other hand, the output perturbation on channel $c$ caused by an input perturbation on the same channel is $\Delta Q_{c}^{\text {out }}(s)=\Delta Q_{c}^{\text {in }}(s) H_{c \text {,self }}(s)$ where the self-filter $H_{c, \text { self }}(s) \triangleq G_{c}\left[1+H_{R}(s) Q_{c}^{\text {in }} B_{c}\left(1-G_{c}\right)\right]$, is either a high pass in the open loop [28], or a notch in the clamped configuration, as shown in Fig. 10. The depth of the notch is approximately $\left\{\left(1 / \tau_{c}\right)-Q_{c} G_{c} B_{c}\right\} /\left\{1 / \tau_{c}\right\}$ [where $\tau_{c}$ is given in (17)], and depends on how large the perturbed channel $c$ is with respect to the others, the laser and the pump. It is usually very small.

\section{A. Chains}

Consider the clamped/open-loop DFA of the previous section followed by a time-invariant loss element, with loss $\left[L_{1} \ldots L_{N}\right]$ for channels 1 through $N$. It is easy to see that the matrix of the DFA+loss system is the same as in (26), with the total gain $G_{j} / L_{j}$ for each channel $j$ used in $\underline{\underline{G}}$ and $Q$ in place

\footnotetext{
${ }^{3} \mathrm{We}$ exclude here the pump from the input perturbations.

${ }^{4} \mathrm{We}$ indicate vectors in boldface and matrices in boldface with double underlining. The symbol ${ }^{T}$ indicates transposition.
} 


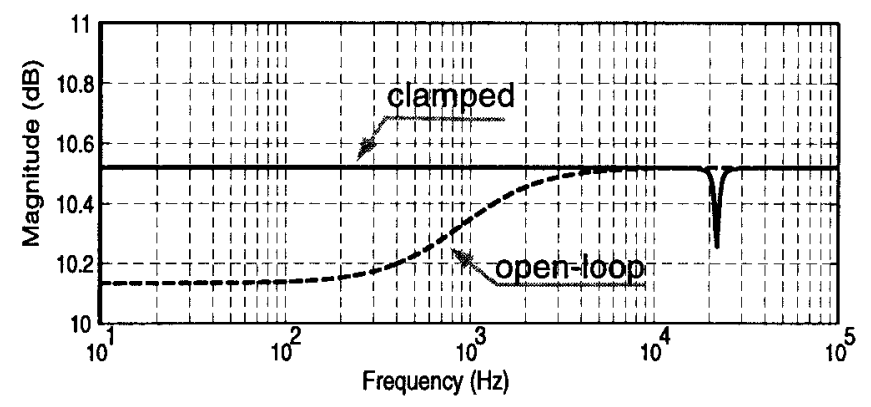

Fig. 10. Self-filter magnitude for clamped and open-loop configurations, same inversion $x=0.6$, eight channels at steady state, same data as in Fig. 6 .
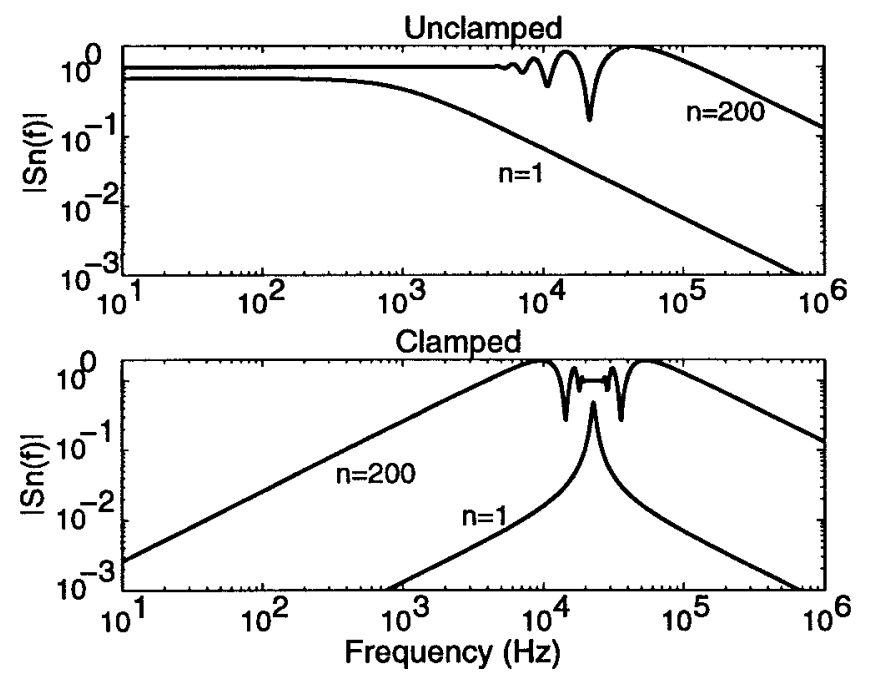

Fig. 11. Equivalent reservoir filter magnitude for a single $(n=1)$ and a chain of $n=200$ DFA+loss modules, both for clamped and open loop configurations, same inversion $x=0.6$, eight channels at steady state, same data as in Fig. 6.

of $G_{j}$. Vector $\boldsymbol{P} \triangleq\left[\left(1-G_{1}\right) \ldots\left(1-G_{N}\right)\right]^{T}$ is unchanged. If a chain is built cascading $n$ copies of the DFA+loss module, the total system matrix is the product of the individual matrices: $\underline{\underline{M}}_{n} \cdots \underline{\underline{M}}_{1}$. Such matrices are different, as the bias points of the modules differ. However, choosing the losses $L_{j} \equiv G_{j}$ in order to perfectly balance the module, we have that the gain matrix becomes the identity matrix $\underline{\underline{G}}=\underline{\underline{I}}$ and all module matrices are equal to $\underline{\boldsymbol{M}}=\underline{\boldsymbol{I}}+H_{R}(s) \overline{\boldsymbol{Q}} \boldsymbol{P}^{T}$, so that the system matrix after $n$ modules is

$$
\underline{\underline{\boldsymbol{M}}}^{n}=\underline{\underline{I}}+\left[(1+S(s))^{n}-1\right] \frac{\boldsymbol{Q} \boldsymbol{P}^{T}}{\left(\boldsymbol{P}^{T} \boldsymbol{Q}\right)}
$$

where we have used the binomial expansion $(\underline{I}+\underline{A})^{n}=$ $\sum_{k=0}^{n}\left(\begin{array}{l}n \\ k\end{array}\right) \underline{\underline{A}}^{k}$ and the fact that $\left(H_{R} \boldsymbol{Q} P^{T}\right)^{k}=H_{R}^{k}\left(\boldsymbol{P}^{T} \boldsymbol{Q}\right)^{k-1}$ $\boldsymbol{Q} \boldsymbol{P}^{T}$ and where

$$
S(s)=\left(\boldsymbol{P}^{T} \boldsymbol{Q}\right) H_{R}(s)
$$

is the reservoir filter normalized by the scalar $\left(\boldsymbol{P}^{T} \boldsymbol{Q}\right)=$ $\Sigma_{j \in \mathcal{S}} Q_{j}^{\text {in }} B_{j}\left(1-G_{j}\right)$. The scalar "equivalent reservoir" filter $S_{n}(s) \triangleq\left[(1+S(s))^{n}-1\right]$ has already been studied in [28] for balanced chains of open-loop (nonclamped) DFA's. In Fig. 11 we give the Bode plot magnitude of such filter, for a single module and a chain of 200 amplifiers, for both clamped and unclamped modules, for the same inversion level. We see that the equivalent reservoir filter for the clamped chain has the high-frequency part of its open-loop counterpart mirrored around the natural frequency $\Omega_{n} /(2 \pi)$. This implies that in a long chain of clamped amplifiers the passband of the equivalent reservoir filter expands to very low frequencies (as well as to very high frequencies), so that the chain becomes more affected by low-frequency disturbances.

\section{SUMMARY AND CONCLUSIONS}

Starting from a simple state-space model of the DFA, we have provided a detailed analysis of both the steady-state and the dynamic behavior of gain-clamped DFA's in a WDM networking environment.

We have provided criteria for the optimal selection of pump power, laser wavelength and laser cavity loss for the design of a gain-clamped DFA with specifications on the number of WDM signals, input signal power per channel, and signal gain. A lower bound on the necessary pump power is given in Fig. 3, and the amount of extra pump needed to satisfy specifications on the maximum overshoot on surviving channels during add-drop operations is given in the contour plots in Fig. 5. From such plots we conclude that the pump power should be chosen 1-2 $\mathrm{dB}$ above its required minimum value. For example, consider a gain-clamped DFA with eight WDM channels, $-3 \mathrm{dBm}$ input signal power per channel, and 12 $\mathrm{dB}$ required signal gain. From the graphs we find that a pump power of $20+1.5=21.5 \mathrm{dBm}$ and a laser placed at $1530 \mathrm{~nm}$ ensure a worst-case overshoot less than $0.25 \mathrm{~dB}$. The required pump value could be obtained using two or more lower power pumps.

While the above plots were derived from the nonlinear model, a linearized analysis lead to simple filters giving the reservoir and output fluxes frequency response to input signal/pump flux perturbations. From these we learned the following:

1) in open-loop DFA, only the low-frequency signal/pump fluctuations are passed to the reservoir and hence to the gain; in clamped DFA's only the fluctuations in a narrow frequency range around the natural system frequency (of the order of some tens of $\mathrm{kHz}$ ) are passed to the reservoir (cf., Fig. 6);

2) long balanced chains of (open-loop) clamped amplifiers have the small-signal behavior of a single (open-loop) clamped amplifier with an "equivalent" reservoir filter of much wider bandwidth, proportional to the number of amplifiers in the chain (cf., Fig. 11);

3) gain-clamped amplifiers can tolerate pump diodes with large low-frequency relative intensity noise (cf., Fig. 6);

4) no low-frequency overtones can be impressed on the channels by modulating the pump; overtones at the natural frequency cannot be used, as this is strongly input-power dependent (cf., Fig. 6);

5) ringing is always present and strong in the natural system response for laser cavity loss above $1 \mathrm{~dB}$ (cf., Fig. 7);

6) the laser wavelength giving minimum overshoot for loss values larger than a few $\mathrm{dBs}$ (hence practical for the loop 
configuration) is the one maximizing the gain slope, i.e., maximizing the total cross-section $\sigma^{e}+\sigma^{a}$, and is usually close to $1530 \mathrm{~nm}$ (cf., Fig. 8);

7) the laser wavelength giving maximum relaxation oscillation damping is the one giving a laser cavity loss very close to 1 (hence interesting for the straight-line configuration, Appendix A), thus having an extremely large laser flux inside the cavity. Such wavelength is close to $1511 \mathrm{~nm}$ (cf., Fig. 8);

8) the extra pump required to satisfy specifications on the maximum overshoot is inversely proportional to the square of the max overshoot, and directly proportional to the number of channels, signal gain and loop delay [Appendix B, (30)];

9) the loop delay $\tau_{l}$ does not affect the decay rate $\Gamma$ but only the natural frequency $\Omega_{n}$ [23];

10) the linear model tends to overestimate the natural frequency, overestimate the reservoir overshoot and underestimate the laser overshoot during add-drops. For our sample DFA, the maximum total input signal power for the linear model to be accurate is $-7 \mathrm{dBm}$ for a pump of $15 \mathrm{dBm}$, and it increases with pump with a slope of $5 \mathrm{~dB} / \mathrm{dec}$ (cf., Fig. 14).

The most serious limitation of the model is its assumption of homogeneous broadening. Placing the laser wavelength too far from the signals may cause offsets in the planned steady-state gain due to spectral hole burning, so that our conclusions on the optimal placement of laser wavelength must be weighed against such inhomogeneous effects [15].

\section{APPENDIX A}

\section{STRAIGHT-Line CONFIGURATION}

This Appendix discusses the straight-line gain-clamped (SLGC) amplifier and gives the necessary modifications to the loop configuration equations.

The SLGC amplifier is composed of a DFA with gratings etched at both DFA ends acting as mirrors, with input and output reflectivity $R_{i}$ and $R_{O}$ at the laser wavelength, and ideally zero reflectivity at signal and pump wavelengths. Indicating with $l^{+}$and $l^{-}$the forward and backward laser fluxes entering the DFA, (1) is still valid with the summation extended over $j \in\left\{\mathcal{S}, p, l^{+}, l^{-}\right\}$. Assuming that a single pass through the DFA takes $\tau_{l} / 2$ seconds, we have $Q_{l^{-}}(t)=$ $Q_{l^{+}}\left(t-\tau_{l} / 2\right) G_{l}\left(r\left(t-\tau_{l} / 2\right)\right) R_{o}$ and $Q_{l^{+}}(t)=Q_{l^{-}}(t-$ $\left.\tau_{l} / 2\right) G_{l}\left(r\left(t-\tau_{l} / 2\right)\right) R_{i}$, so that

$$
Q_{l^{+}}(t)=\alpha\left\{Q_{l^{+}}\left(t-\tau_{l}\right) G_{l}\left(r\left(t-\tau_{l}\right)\right) G_{l}\left(r\left(t-\tau_{l} / 2\right)\right)\right\}
$$

where $\alpha \triangleq R_{i} R_{o}$ is the roundtrip attenuation. This should be compared to (2) for the loop configuration. The Barkhausen

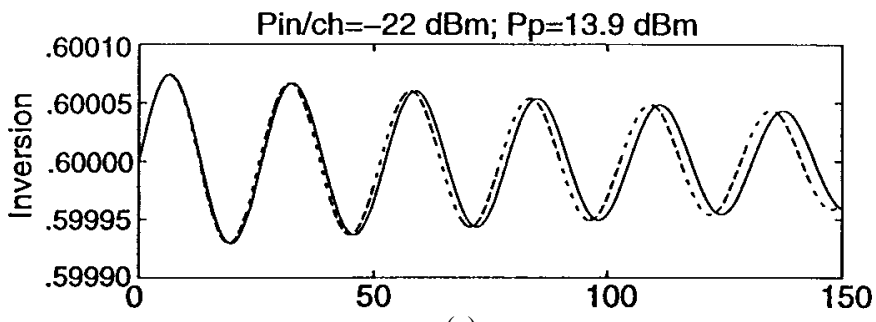

(a)

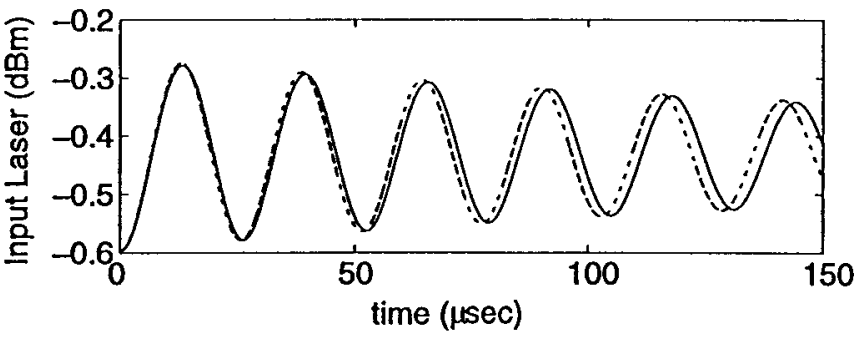

(b)

Fig. 12. (a) Normalized reservoir and (b) input laser power dynamic step response to a drop of seven out of eight channels; small-signal solution (solid) and exact solution (dashed). Data: $P_{p}=13.9 \mathrm{dBm}, P_{\mathrm{in} / \mathrm{ch}}=-22 \mathrm{dBm}$, $x=0.6 ; \lambda_{l}=1530 \mathrm{~nm}, \tau_{l}=0.18 \mu \mathrm{s}$.

criterion is here $G_{l}^{s s}=\sqrt{1 / \alpha}$, and the steady-state reservoir is $r_{s s}=\left(\frac{1}{2} \ln (1 / \alpha)+A_{l}\right) / B_{l}$, while the steady-state net laser flux is

$$
Q_{l^{+}}^{s s}=\frac{\sum_{j \in\{\mathcal{S}, p\}} Q_{j}\left[1-G_{j}^{s s}\right]-\frac{r_{s s}}{\tau}-Q_{\mathrm{ASE}}\left(r_{s s}\right)}{\left(\sqrt{\frac{1}{\alpha}}-1\right)\left(1+\sqrt{\frac{R_{o}}{R_{i}}}\right)} .
$$

Here, the round-trip loss can be very small and thus $Q_{l^{+}}^{s s}$ very large. For instance, if $R_{i}=0.99$ and $R_{o}=0.92$ we have a round-trip loss of $0.45 \mathrm{~dB}$. Therefore, in the straightline configuration a laser wavelength around $1511 \mathrm{~nm}$ should be feasible, with the advantage of a good dynamic behavior as shown in Fig. 9. Following the approach in the text, the clamped reservoir filter can be calculated to be the equation shown at the bottom of the page where $1 / \tau_{c}$ is as in (17) with the summation extended to $j \in\left\{\mathcal{S}, p, l^{+}, l^{-}\right\}$, and $\tau_{l}$ is almost twice the round-trip delay in the loop configuration.

\section{APPENDIX B \\ ACCURACY OF LINEAR MODEL}

To check the accuracy of the linear model, in Fig. 12 the small-signal reservoir and input laser power obtained as per (20) and (19) are shown in solid line and compared to the exact solution of (1) and (2), in dashed lines, when seven out of the eight WDM channels are dropped. The input power per channel, $-22 \mathrm{dBm}$, is small enough that the linear

$$
H_{c}(s)=\frac{s\left(1+\frac{\tau_{l}}{4} s\right)^{2}}{\left(s+\frac{1}{\tau_{c}}\right) s\left(1+\frac{\tau_{l}}{4} s\right)^{2}+\left(\sqrt{\frac{1}{\alpha}}-1\right)\left(1+\frac{\tau_{l}}{4} s+\sqrt{\frac{R_{o}}{R_{i}}}\left(1-\frac{\tau_{l}}{4} s\right)\right) \frac{2 Q_{l^{+}}^{s} B_{l}}{\tau_{l}}\left(1-\frac{\left(\tau_{l} s\right)^{2}}{8}\right)}
$$


Pinch $=-10 \mathrm{dBm} ; \mathrm{PP}=13.9 \mathrm{dBm}$

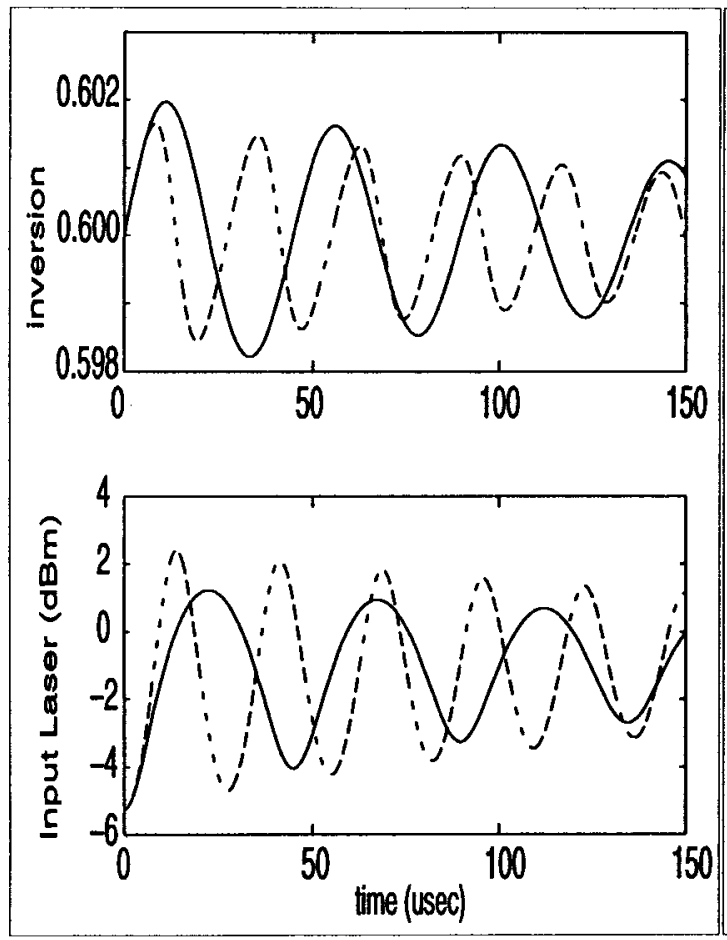

(a)

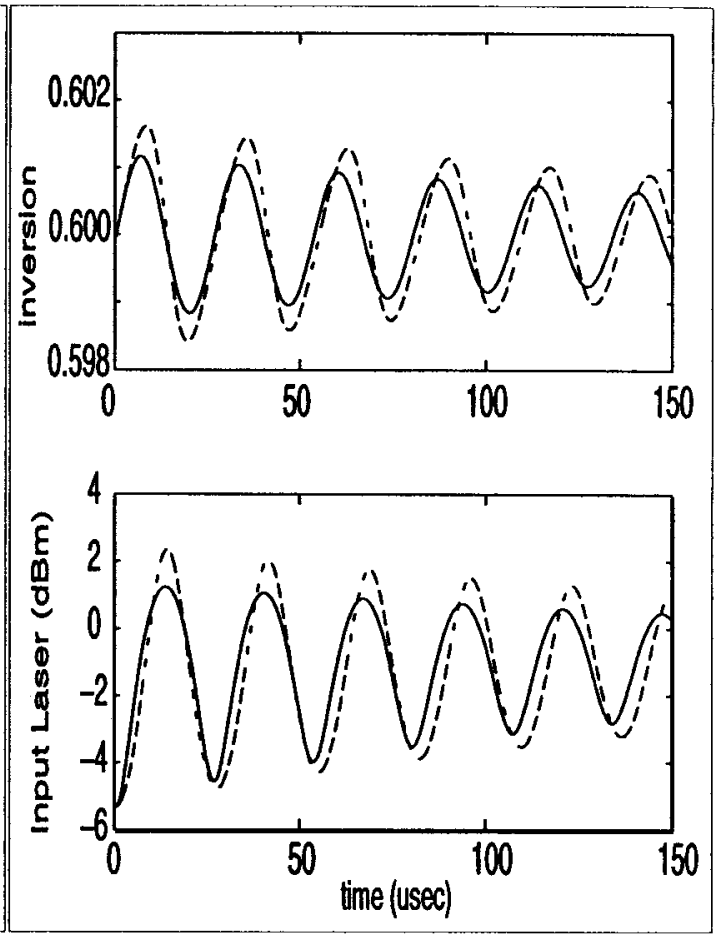

(b)

Fig. 13. (a) Same as in Fig. 12, but with larger input power per channel of $-10 \mathrm{dBm}$. (b) Same as (a), but using in the theoretical model the steady-state values $Q_{j}^{s s}$ reached after the step.

approximation is fairly good, with a slight overestimation of the relaxation frequency $\Omega$, the accuracy improving for lower input powers.

Fig. 13(a) shows the same transient with a larger input power of $-10 \mathrm{dBm}$, with a more evident underestimation of $\Omega$ and consequent overestimation of the reservoir overshoot and an underestimation of the laser overshoot. Fig. 13(b) shows how to improve the accuracy of the linear model for add-drops: it is enough to use the steady state signal and laser fluxes $Q_{j}^{s s}, j \in\{\mathcal{S}, l\}$, after the step instead of before it in (17), (18). This also explains why in the nonlinear regime the relaxation oscillation frequency is larger after drops than after adds: the subsequent steady-state laser level is larger after the drops, and so is $\Omega_{n}$ as per (18), while decay rates, as seen in (23), are comparable.

We have verified that the linear model is fairly accurate (cf., Fig. 12) when $B_{\max } \Delta r_{\max } \leq 0.01$, where $B_{\max }=$ $\max _{j \in\{\mathcal{S}, p, l\}}\left\{B_{j}\right\}$. To express such inequality as a bound on input power, we assume equal signal fluxes and gains: $Q_{j} \equiv Q_{s}, G_{j} \equiv G_{s}, \forall j \in \mathcal{S}$; we approximate $G_{s}-1 \cong$ $G_{s}$, and consider the worst-case drop of $N-1$ out of $N$ channels, so that $|\mathcal{K}| \cong(N-1) Q_{s} G_{s}$. Using (22), (18), and (6) the above inequality becomes a parabola in $Q_{s}$ : $A Q_{s}^{2}+B Q_{s}+C \leq 0$, where $A=\left(100 B_{\max }(N-1) G_{s}\right)^{2}$; $B=\left(B_{l} / \tau_{l}\right) N G_{s} ; C=-\left(B_{l} / \tau_{l}\right)\left[Q_{p}\left(1-G_{p}^{s s}\right)-\left(r_{s s} / \tau\right)\right]$. Fig. 14 shows in bold line such upper bound on signal power $P_{s}=h \nu Q_{s}$ versus pump power, for $N=8,16,32$, and the corresponding laser power as in (6). Dashed lines correspond to the following approximations for large pump $Q_{p}: Q_{s} \cong$

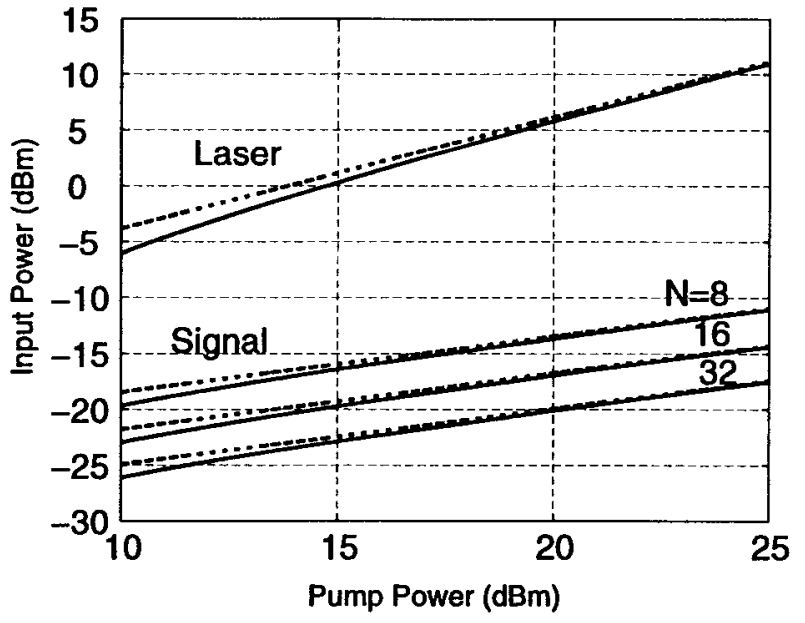

Fig. 14. Maximum input power per channel $P_{S}$ and corresponding input laser power $P_{l}$ for linear model to be accurate, versus pump power. Number of WDM channels $N$ as a parameter. Solid: parabolic formula; dashed: approximation. Data: signal gain $G_{s}=10.72 \mathrm{~dB}(x=0.6) ; \tau_{l}=0.18 \mu \mathrm{s}$; $\lambda_{l}=1530 \mathrm{~nm}$.

$\sqrt{\left(B_{l} / \tau_{l}\right) Q_{p}\left(1-G_{p}^{s s}\right)} /\left(100 B_{\max }(N-1) G_{s}\right), Q_{l}^{s s} \cong Q_{p}(1-$ $\left.G_{p}^{s s}\right) /(1 / \alpha-1)$. Note the $5 \mathrm{~dB} /$ decade slope of the signal power and its linear dependence on $N$, and the $10 \mathrm{~dB} /$ decade slope of laser power, independent of $N$.

We conclude this Appendix with an analytical smallsignal approximation of the contour curves of maximum dB-excursion on surviving channel $s$ given in Fig. 5. At high resonance, from (21) and (22) we have $\epsilon_{\max } \cong 4.34 B_{s}|\mathcal{K}| / \Omega_{n}$ 
$(\mathrm{dB})$, the approximation being excellent for $\epsilon_{\max } \leq 4.34 *$ $0.01=0.0434 \mathrm{~dB}$. We assume as before equal signal fluxes $Q_{s}$ and large gains $G_{s}$, so that $|\mathcal{K}| \cong(N-1) Q_{s} G_{s}$. Using $\Omega_{n}$ as in (23) and approximating $(N-1) Q_{s} G_{s} \cong Q_{p}^{L}\left(1-G_{p}\right)$ for large $N$ we get:

$$
\frac{Q_{p}}{Q_{p}^{L}} \cong 1+\frac{\tau_{l}}{B_{l}} N Q_{s} G_{s}\left(\frac{4.34 B_{s}}{\epsilon_{\max }}\right)^{2} .
$$

For values of $\epsilon_{\max }>0.1 \mathrm{~dB}$ such expression always overestimates the required pump $Q_{p}$. Nontheless, (30) is useful to highlight the linear dependence on output signal flux $Q_{s} G_{s}$, on channel number $N$, on loop delay $\tau_{l}$; the inverse dependence on $B_{l}$ and the inverse quadratic dependence on $\epsilon_{\max }$.

\section{ACKNOWLEDGMENT}

The authors gratefully acknowledge Prof. A. Piazzi for suggesting the Padé approximation of the delay term. A. Bononi wishes to thank Prof. L. Rusch for rekindling his interest in optical amplifiers.

\section{REFERENCES}

[1] K. Motoshima, L. M. Leba, D. N. Chen, M. Downs, T. Li, and E. Desurvire, "Dynamic compensation of transient gain saturation in erbium-doped fiber amplifiers by pump feedback control," IEEE Photon. Technol. Lett., vol. 5, pp. 1423-1426, Dec. 1993.

[2] N. Takahashi, T. Hirono, H. Akashi, S. Takahashi, and T. Sasaki, "An outout power stabilized erbium-doped fiber amplifier with automatic gain control," J. Select. Topics Quantum Electron., vol. 3, pp. 1019-1026, Aug. 1997.

[3] S. Y. Park, H. K. Kim, G. Y. Lyu, S. M. Kang, and S.-Y. Shin, "Dynamic gain and output power control in a gain-flattened erbium-doped fiber amplifier," IEEE Photon. Technol. Lett., vol. 10, pp. 787-789, June 1998.

[4] L. Tancevski, L. A. Rusch, and A. Bononi, "Gain control in EDFA's by pump compensation," IEEE Photon. Technol. Lett., vol. 10, no. 9, Sept. 1998

[5] J. L. Zyskind, A. K. Srivastava, Y. Sun, J. C. Ellson, G. W. Newsome, R. W. Tkach, A. R. Chraplyvy, J. W. Sulhoff, T. A. Strasser, J. R. Pedrazzani, and C. Wolf, "Fast link control protection for surviving channels in multiwavelength optical networks," in Proc. ECOC '96, Oslo, Norway, 1996, vol. 5, pp. 49-52.

[6] M. Zirngibl, "Gain control in erbium-doped fiber amplifiers by an alloptical feedback loop," Inst. Elec. Eng. Electron. Lett., vol. 27, pp. 560-561, 1991.

[7] E. Delevaque, T. Georges, J. F. Bayon, M. Monerie, P. Niay, and P. Berage, "Gain control in erbium-doped fiber amplifiers by lasing at 1480 $\mathrm{nm}$ with photoinduced Bragg gratings written on the fiber ends," Inst. Elec. Eng. Electron. Lett., vol. 29, pp. 1112-1114, 1993.

[8] J. F. Massicott, S. D. Wilson, R. Wyatt, J. R. Armitage, R. Kashyap, and D. Williams, "1480 nm pumped erbium doped fiber amplifier with all-optical automatic gain control," Inst. Elec. Eng. Electron. Lett., vol. 30, pp. 962-964, 1994.

[9] J. C. Simon, P. Doussiere, P. Lamouler, I. Valiente, and F. Riou, "Travelling wave semiconductor optical amplifier with reduced nonlinear distortions," Inst. Elec. Eng. Electron. Lett., Jan. 1994, pp. 49-50.

[10] G. Soulage, A. Jourdan, P. Doussiere, and G. da Loura, "Clamped-gain SOA gates as multiwavelength space switches," in Proc. OFC '95, San Diego, CA, Feb. 1995, pp. 9-10.

[11] R. Lebref, B. Landousies, T. Georges, and E. Delevaque, "Study of power transients in EDFA with gain stabilization by a laser effect," Inst. Elec. Eng. Electron. Lett., vol. 33, pp. 191-193, Jan. 1997.

[12] D. H. Richards, M. A. Ali, and J. L. Jackel, "A theoretical investigation of dynamic automatic gain control in multichannel EDFA's and EDFA cascades," IEEE J. Select. Areas Quantum Electron., vol. 3, pp. 1027-1036, Aug. 1997.

[13] J. Chung and S. Y. Kim, "Dynamic performance of the all-optical gaincontrolled EDFA cascade in multi-wavelength add/drop networks," in Proc. ECOC '97, Edinburgh, U.K., Sept. 1997, pp. 139-142.
[14] G. Luo, J. L. Zyskind, Y. Sun, A. K. Srivastava, J. W. Sulhoff, C. Wolf, and M. A. Ali, "Performance degradation of all-optical gainclamped EDFA's due to relaxation oscillations and spectral hole burning in amplified WDM networks," IEEE Photon. Technol. Lett., vol. 9, pp. 1346-1348, Oct. 1997.

[15] G. Luo, J. L. Zyskind, J. A. Nagel, and M. A. Ali, "Experimental and theoretical analysis of relaxation-oscillations and spectral hole burning effects in all-optical gain-clamped EDFA's for WDM networks," $J$. Lightwave Technol., vol. 16, pp. 527-533, Apr. 1998.

[16] A. Bononi and L. A. Rusch, "Doped fiber amplifier dynamics: A system perspective," J. Lightwave Technol., vol. 16, pp. 945-956, May 1998.

[17] A. Bononi, L. Barbieri, and L. A. Rusch, "Using SPICE to simulate gain dynamics in doped-fiber amplifier chains," presented at OFC '98 Workshop 204 Transmission Modeling Simulation Tools, San Jose, CA, Feb. 23, 1998

[18] T. Georges and E. Delevaque, "Analytic modeling of high-gain erbiumdoped fiber amplifiers," Opt. Lett., vol. 17, pp. 1113-1115, Aug. 1992.

[19] J. Millman, Microelectronics. New York: McGraw-Hill, 1979, ch. 14

[20] A. R. Hambley, Electronics. New York: MacMillan, 1994, ch. 8.

[21] A. Yu and M. J. O'Mahony, "Design and modeling of laser-controlled erbium-doped fiber amplifiers," J. Select. Topics Quantum Electron., vol. 3, pp. 1013-1018, Aug. 1997.

[22] E. Desurvire, Erbium-Doped Fiber Amplifiers. New York: Wiley, 1994.

[23] R. Lebref, B. Landousies, T. Georges, and E. Delevaque, "Theoretical study of the gain equalization of a stabilized gain EDFA for WDM applications," J. Lightwave Technol., vol. 15, pp. 766-770, May 1997.

[24] A. E. Siegman, Lasers. New York: University Science Books, 1986

[25] Y. Sun, A. A. M. Saleh, J. L. Zyskind, D. L. Wilson, A. K. Srivastava, and J. W. Sulhoff, "Time dependent perturbation theory and tones in cascaded erbium-doped fiber amplifier systems," J. Lightwave Technol., vol. 15, pp. 1083-1087, July 1997.

[26] G. F. Franklin, J. D. Powell, and A. Emami-Naeini, Feedback Control of Dynamic Systems. Reading, MA: Addison Wesley, 1991.

[27] M. Cai, X. Liu, J. Cui, P. Tang, and J. Peng, "Study on noise characteristic of gain-clamped erbium-doped fiber-ring lasing amplifier," IEEE Photon. Technol. Lett., vol. 9, pp. 1093-1095, Aug. 1997.

[28] A. Mecozzi and D. Marcenac, "Theory of optical amplifier chains," $J$. Lightwave Technol., vol. 16, pp. 745-756, May 1998.

Alberto Bononi was born in La Spezia, Italy, on December 7, 1963. He received the Dr.Ing. degree (cum laude) in electronics engineering from the University of Pisa, Pisa, Italy, in 1988 and the M.A. and Ph.D degrees in electrical engineering from Princeton University, Princeton, NJ, in 1992 and 1994, respectively.

In 1990, he worked at GEC-Marconi Hirst Research Centre, Wembley, U.K., on a Marconi S.p.A. Project on coherent optical FSK systems. From 1994 to 1996, he was an Assistant Professor in the Electrical and Computer Engineering Department at the State Universityof New York (SUNY), Buffalo, NY, teaching courses on Electric Circuits and on Optical Networks. Since 1996, he has been an Assistant Professor at the Universita' di Parma, Italy. He teaches a course on Telecommunications Networks for the fifth year students of Telecommunications Enginering, and a course on signals, probability theory and stochastic processes for the second year students of Telecommunications, Information and Electrical Engineering. His current research interests include system design and performance issues in fast packet switching and highspeed all-optical networks; nonlinear fiber transmission for WDM systems; and transient gain dynamics in doped-fiber amplifiers. From 1996 to 1998, he was the Scientific Coordinator of the European Community sponsored project DAWRON (design of advanced wavelength routed optical networks).

Lorenzo Barbieri was born in Reggio Emilia, Italy, on November 6, 1973. $\mathrm{He}$ received the Dr.Ing. degree (cum laude) in electronics engineering from the University of Parma, Parma, Italy, in April 1998.

His research interests include transient gain dynamics in doped-fiber amplifiers and the study of Kerr nonlinearities in high bit rate multiwavelength communications systems. 